\title{
Effect of Lifestyle Interventions in Obese Pregnant Women on the Neurocognitive Development and Anthropometrics of Preschool Children
}

\author{
Marijke Anne Katrien Alberta Braeken ${ }^{a, b} \quad$ Annick Bogaerts ${ }^{c, d}$ \\ ${ }^{a}$ Research Unit Resilient People, Faculty of Health and Social Work, University Colleges \\ Leuven-Limburg, Diepenbeek, Belgium; ${ }^{\text {b}}$ Rehabilitation Research Center, Biomedical \\ Research Institute, Faculty of Rehabilitation Sciences, Hasselt University, Hasselt, Belgium; \\ 'Department of Development and Regeneration, KU Leuven, Leuven, Belgium; ${ }^{\mathrm{d}}$ Faculty of \\ Medicine and Health Sciences, Centre for Research and Innovation in Care (CRIC), University \\ of Antwerp, Antwerp, Belgium
}

\section{Keywords}

Lifestyle intervention · Pregnancy · Offspring development

\begin{abstract}
Introduction: Maternal obesity and excessive gestational weight gain are related to adverse outcomes in women and children. Lifestyle interventions during pregnancy showed positive effects on decreasing weight gain during pregnancy, but effects on offspring's health and wellbeing are unclear. We aimed to assess the effect of lifestyle intervention programmes on offspring mental health, temperament, eating habits and anthropometric and cardiovascular measures. Methods: Ninety-six offspring of pregnant women with a body mass index (BMI) $\geq 29 \mathrm{~kg} / \mathrm{m}^{2}$ who were randomly assigned to 3 intervention groups during pregnancy (routine antenatal care, a brochure group or a prenatal session group) and 77 offspring of pregnant women with a normal BMI (between 18.5 and $24.9 \mathrm{~kg} / \mathrm{m}^{2}$ ) were used as an additional control group in this analysis. When the children were between 3 and 7 years old, anthropometric and cardiovascular measurements were conducted and various questionnaires about offspring mental health, temperament and eating habits were filled out. Results: Children of mothers who received a brochure-based lifestyle intervention programme showed significantly less surgency/extraversion compared to children of mothers who received routine antenatal care (contrast estimate $=-0.36, \mathrm{SE}=0.15, p=0.02,95 \% \mathrm{Cl}[-6.66,-0.06]$ ) and prenatal lifestyle intervention sessions (contrast estimate $=-0.46, \mathrm{SE}=0.14, p<0.01,95 \% \mathrm{Cl}[-0.74,-0.18]$ ) after adjusting for child's age, sex, offspring birth weight and mother's educational level. The lifestyle intervention could not be associated with any significant differences in offspring
\end{abstract}


Braeken and Bogaerts: Effect of Lifestyle Interventions in Obese Pregnant Women on the Neurocognitive Development and Anthropometrics of Preschool Children

mental health, eating habits and anthropometric and cardiovascular characteristics. Children of mothers with a normal BMI showed less emotional problems $(F(1,156)=5.42, p=0.02)$ and internalizing $(F(1,156)=3.04, p=0.08)$ and externalizing problems $(F(1,156)=6.10, p=0.02)$ when compared to children of mothers in the obese group. Discussion/Conclusion: The results suggest that a brochure-based lifestyle intervention programme can affect the offspring temperament. Future follow-up studies need to investigate how these temperament-related effects may influence obesity development later in life.

(C) 2020 The Author(s)

Published by S. Karger AG, Basel

\section{Introduction}

The past 30 years have seen an exponential increase in global rates of obesity in all population groups, including pregnant women and children, recognized worldwide as a major burden for public health. Estimates for maternal obesity during pregnancy from 23 European countries suggest that the prevalence varies from 7 to 25\% [1]. Demographic variation in the incidence of obesity is clearly evident [2], with the prevalence of maternal obesity being strongly related to social, educational and ethnic differences [3]. The prevalence of overweight and obesity in European children (aged 2-7 years) during the period 2006-2016 was $17.9 \%$ [4]; and worldwide, 41 million children under the age of 5 years were overweight or obese in 2016 [5]. Furthermore, the prevalence of overweight and obesity among children and adolescents aged 5-19 years has risen dramatically from just 4\% in 1975 to just over $18 \%$ in 2016 [3, 6-9].

Maternal overweight and obesity is linked to increased offspring's risk of obesity and has shown to be of stronger influence than either gestational weight gain (GWG) or postpartum weight retention [9]. Additionally, GWG is positively associated with offspring adiposity [7, 10] and an increased risk for childhood cardiovascular risk profile [11]. A recent metaanalysis showed that offspring of pregnant women who gained excess weight during pregnancy had $40 \%$ increased risk of offspring obesity from early to late adolescence and adulthood [12]. Evidence suggests that associations between pre-pregnancy obesity and early excessive GWG with adverse cardiometabolic profiles in offspring are largely mediated by offspring's childhood body mass index (BMI) [11].

Concurrently, maternal obesity can also lead to poorer cognitive performance and increased risk of neurodevelopmental disorders in their offspring [8]. Some human studies have shown that higher pre-pregnancy BMI is associated with poorer cognitive development and emotional problems in offspring $[13,14]$. However, published data do not allow for definitive conclusions due to methodological limitations. Most studies are longitudinal observational cohorts faced with residual confounders. Mediating and moderating variables, such as breastfeeding, paternal BMI, eating behaviour within a family context and mental health and temperament of the children, are mostly not taken into account. Previous studies report on associations between difficult temperament and risk for eating and weight regulation problems in later childhood [15].

Worryingly, $20-40 \%$ of obese children and adolescents are diagnosed with neurodevelopmental disorders. For instance, attention deficit disorder and autism spectrum disorders are almost twice as high in obese compared to non-obese school-age children. Executive dysfunction, perception memory, language and learning problems, reduced social skills and emotional instability are 2-4 times higher and motor skills disorder 5 times higher in obese children. Clearly, these neurodevelopmental disorders require the same attention as the prevention of childhood obesity [16]. 
Braeken and Bogaerts: Effect of Lifestyle Interventions in Obese Pregnant Women on the Neurocognitive Development and Anthropometrics of Preschool Children

Nutrient intake and weight gain during pregnancy are 2 main modifiable factors influencing maternal and infant outcomes. Most intervention studies during pregnancy have focused on the limitation of GWG as a primary outcome by improving diet and physical activity. Unfortunately, other than decreasing GWG by 1-2 kg, these studies have not improved perinatal outcomes, including fetal overgrowth [17-19]. The LIMIT study, however, a randomized controlled trial (RCT) $(n=1,108)$ powered for clinical outcomes of the child, has shown a significant decrease in women giving birth to macrosomic infants (15 vs. 19\%, number needed to treat $=28, p=0.04$ ) and suggests that lifestyle interventions during pregnancy (including dietary, exercise and behavioural strategies) might be effective in modulating the offspring's outcome [20].

A recently published unique individual patient data meta-analysis of 12,526 pregnant women across 16 countries worldwide found that diet- combined with physical activitybased interventions significantly reduced the mother's weight gain during pregnancy by an average of $0.7 \mathrm{~kg}$ compared to the control group and lowered the risk of the mother having a caesarean delivery by about $10 \%$; however, no further maternal and neonatal composite outcomes were significant [21]. Whether the observed benefit in GWG with diet and physical activity translates to long-term benefits to the mother and child needs to be assessed. Studies on long-term effectiveness of these lifestyle interventions and the magnitude of weight reduction in relation to significant health and wellbeing benefits for the child need to be conducted [22]. Therefore, the aim of this follow-up study of a randomized controlled lifestyle intervention during pregnancy in obese women is to assess the effect on childhood anthropometrics, offspring eating habits and neurocognitive development in the 3- to 7-year-old offspring. Additionally, differences in cardiovascular measures, such as heart rate and blood pressure, between the intervention groups are studied. A secondary aim is to assess differences in anthropometrics, neurocognitive development, eating habits and cardiovascular outcomes between children of normal-weight versus obese women.

\section{Materials and Methods}

\section{Participants and Procedure}

In an earlier study, 205 obese pregnant women with a BMI $\geq 29 \mathrm{~kg} / \mathrm{m}^{2}$ (obese group) and 208 pregnant women with a normal BMI (between 18.5 and $24.9 \mathrm{~kg} / \mathrm{m}^{2}$, control group) were recruited in 3 regional hospitals between 2008 and 2011. The present article describes a follow-up study in which 96 mothers and their offspring from the obese group and 77 mothers and their offspring from the control group participated (Fig. 1).

Obese pregnant women attending the antenatal clinic before 15 weeks of pregnancy were informed by their gynaecologist or midwife about the study. They introduced the study and gave the women an information sheet about the trial to be read at home. In case of interest, the research midwife contacted the obese pregnant women by phone as soon as possible with the demand to participate. After having given their written informed consent and having completed a baseline assessment on socio-demographics and lifestyle data, as well as on detailed medical and obstetrical history, they were randomly assigned to 3 intervention groups: either (1) a group receiving routine antenatal care, (2) a brochure group receiving besides the routine antenatal care - additionally a purpose-designed brochure about nutritional advice and physical activity during pregnancy with information to limit excessive GWG, or (3) a prenatal session group, receiving routine antenatal care, the same brochure and an additional 4 prenatal lifestyle intervention sessions led by a midwife trained in motivational lifestyle intervention (A.B.). The primary outcomes of this trial (ClinicalTrials.gov Identifier: NCT01329835) in obese pregnant women were that women receiving the brochure-based 
Braeken and Bogaerts: Effect of Lifestyle Interventions in Obese Pregnant Women on the Neurocognitive Development and Anthropometrics of Preschool Children

\section{Bogaerts}

et al. (2013)

\section{This study}

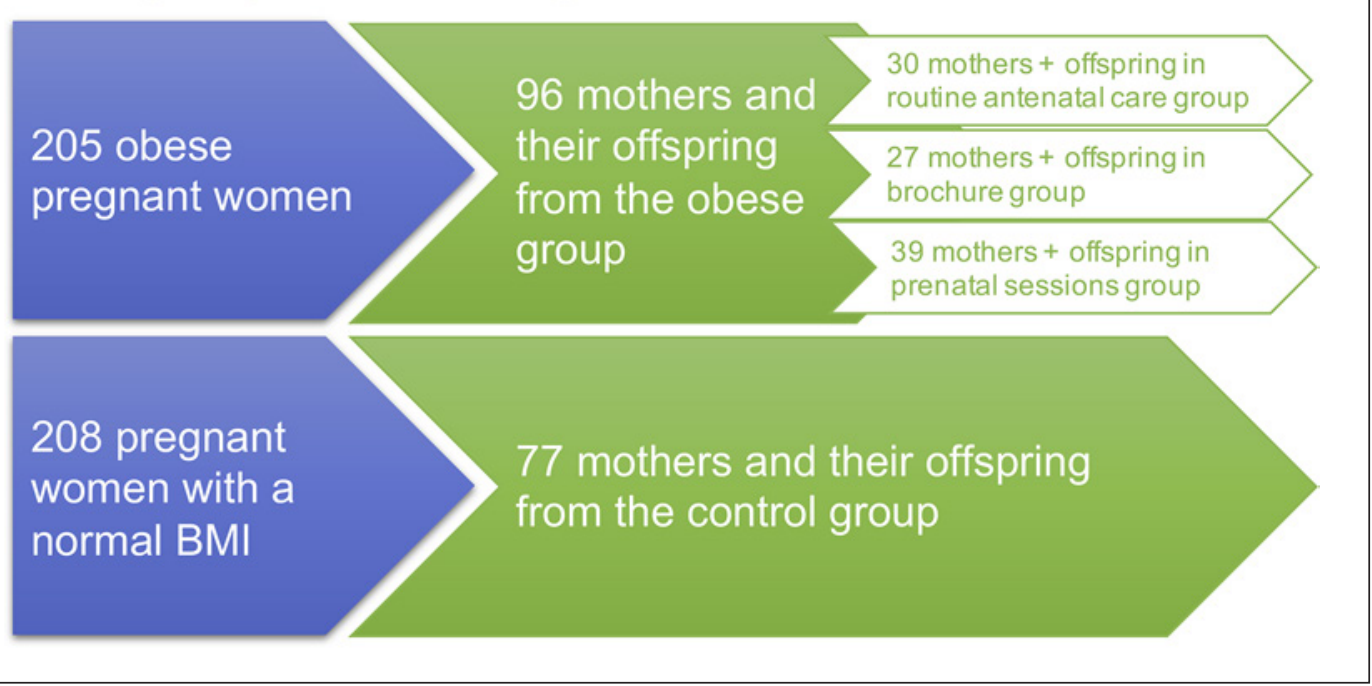

Fig. 1. Flow chart of the sample size in Bogaerts et al. [23] and this study.

lifestyle intervention programmes and the face-to-face lifestyle intervention demonstrated improvements in less GWG [23]. Secondly, obese women in the face-to-face lifestyle intervention group demonstrated lower levels of maternal anxiety during pregnancy [23].

When the children were between 3 and 7 years old, mothers were contacted again to ask whether we could visit them at home to conduct anthropometric and cardiovascular measurements and let them fill out various questionnaires.

\section{Materials}

Child Measurements

The Strengths and Difficulties Questionnaire (SDQ) designed by Goodman [24] was used to measure the offspring's mental health. The SDQ consists of 25 items divided into 5 scales of 5 items each: emotional problems, conduct problems, hyperactivity, peer problems and prosocial behaviour. Each item needs to be scored by the mother on a 3-point scale $(0=$ not true, 1 = somewhat true, and 2 = certainly true). Item scores are summed up to obtain scale scores. Furthermore, a total difficulties score can be calculated by summing the scores on the emotional problems, conduct problems, hyperactivity and peer problems scales. Finally, internalizing and externalizing problems scores can be constructed by adding, respectively, the emotional and peer problems scores, and the conduct problems and hyperactivity scores together. The Dutch version of the SDQ [25] was used and filled out by the mothers.

The offspring temperament was measured using the Dutch version of the Children's Behavior Questionnaire (CBQ) [26], also filled out by the mother. The CBQ is typically used in early to middle childhood and consists of 36 items, each rated on a 7-point Likert scale. Three factors have been reliably recovered from this instrument, labelled negative affectivity, surgency/extraversion and effortful control.

Height and weight were measured by the researchers when visiting the mother's home, and BMI was calculated. The weight was measured using a portable, electronic weighing scale (class 3, for medical use). It has a tare facility and weighs in kilograms to the nearest $50 \mathrm{~g}$. A 
Braeken and Bogaerts: Effect of Lifestyle Interventions in Obese Pregnant Women on the Neurocognitive Development and Anthropometrics of Preschool Children

Table 1. Maternal characteristics

\begin{tabular}{|c|c|c|c|c|c|c|c|c|}
\hline & \multicolumn{2}{|c|}{$\begin{array}{l}\text { Normal-weight } \\
\text { control group }\end{array}$} & \multicolumn{2}{|c|}{$\begin{array}{l}\text { Routine } \\
\text { antenatal care }\end{array}$} & \multicolumn{2}{|c|}{ Brochure } & \multicolumn{2}{|c|}{$\begin{array}{l}\text { Prenatal } \\
\text { sessions }\end{array}$} \\
\hline & mean & SD & mean & SD & mean & SD & mean & SD \\
\hline Age, years & 28.97 & 3.70 & 28.67 & 3.68 & 29.89 & 4.19 & 28.46 & 3.73 \\
\hline Height, $\mathrm{cm}$ & 165.68 & 5.80 & 166.06 & 6.45 & 164.17 & 5.85 & 166.14 & 5.45 \\
\hline Weight, kg & 64.72 & 6.34 & 100.70 & 18.72 & 89.26 & 20.43 & 96.36 & 14.82 \\
\hline BMI & 23.61 & 2.40 & 36.41 & 5.44 & 33.09 & 7.34 & 34.94 & 5.25 \\
\hline Circumference at umbilicus level, cm & 80.74 & 7.20 & 109.76 & 15.76 & 104.25 & 12.54 & 105.05 & 12.08 \\
\hline Heart rate, bpm & 71.07 & 9.90 & 74.90 & 6.55 & 74.83 & 10.11 & 76.89 & 12.30 \\
\hline Diastolic blood pressure, $\mathrm{mm} \mathrm{Hg}$ & 71.68 & 8.73 & 81.44 & 8.99 & 79.97 & 12.63 & 76.35 & 9.20 \\
\hline Systolic blood pressure, $\mathrm{mm} \mathrm{Hg}$ & 117.71 & 13.82 & 128.92 & 16.45 & 128.01 & 14.55 & 122.37 & 12.38 \\
\hline Weekly consumed candy servings & 3.63 & 3.15 & 3.35 & 3.41 & 2.73 & 2.15 & 3.49 & 2.03 \\
\hline Weekly consumed meat servings & 4.58 & 2.00 & 4.60 & 1.77 & 4.52 & 1.95 & 4.86 & 2.48 \\
\hline Weekly consumed fish servings & 0.97 & 0.61 & 1.27 & 0.88 & 1.46 & 0.86 & 1.26 & 0.65 \\
\hline Weekly consumed milk servings & 5.45 & 3.40 & 6.77 & 5.26 & 6.78 & 5.84 & 5.78 & 3.44 \\
\hline Weekly consumed fruit servings & 7.09 & 4.32 & 5.70 & 3.81 & 7.48 & 6.20 & 6.32 & 5.24 \\
\hline Weekly consumed vegetables servings & 6.63 & 2.54 & 7.08 & 2.18 & 7.00 & 2.29 & 6.86 & 2.34 \\
\hline Weekly consumed soft drink servings & 0.83 & 1.02 & 1.35 & 2.04 & 1.29 & 1.11 & 0.98 & 1.36 \\
\hline Weekly consumed water servings & 5.17 & 3.48 & 6.28 & 2.75 & 5.46 & 4.90 & 5.88 & 2.82 \\
\hline
\end{tabular}

Seca Stadiometer with an accuracy of $0.1 \mathrm{~cm}$ was used to measure height. Furthermore, hip and waist circumferences and circumference at the umbilicus level were measured, allowing to compute waist-to-hip and waist-to-height ratios. Additionally, skinfolds thicknesses were measured at 4 locations (triceps, biceps, subscapular and suprailiac) using holtain calipers (Harpenden ${ }^{\circledR}$ Skinfold Cap meter, with an accuracy of $1 \mathrm{~mm}$ ). These skinfolds measurements were summed into a measure of total body fat.

At the end of our visit, heart rate and both diastolic and systolic blood pressure were measured with an OMRON monitor (Omron Healthcare Co., Ltd., Kyoto, Japan). Mothers also filled in a non-standardised questionnaire to estimate the number of weekly consumed servings of candy, meat, fish, milk, fruit, vegetables, soft drink and water by mother and child.

Maternal Measurements

During the visit, height and weight were measured, and BMI was calculated. Furthermore, circumference at the umbilicus level was measured. At the end of our visit, heart rate and both diastolic and systolic blood pressure were measured. For these measurements, the same devices were used as for the child.

\section{Paternal Measurements}

Fathers filled in the same food questionnaire as described above. Furthermore, all fathers filled in a questionnaire about their educational level, weight and height.

\section{Statistical Analysis}

Statistical analyses were performed using SPSS 24. First, one-way multivariate analysis of covariance (MANCOVA) was conducted to compare the effects of the 3 different lifestyle interventions during pregnancy on offspring mental health, temperament, eating habits, anthropometric and cardiovascular measures, while controlling for the children's age and sex, offspring birth weight and the mothers' educational level. Planned contrasts were performed to compare the routine antenatal care group to the brochure group, and to compare the brochure group 
Braeken and Bogaerts: Effect of Lifestyle Interventions in Obese Pregnant Women on the Neurocognitive Development and Anthropometrics of Preschool Children

Table 2. Paternal characteristics

\begin{tabular}{|c|c|c|c|c|c|c|c|c|}
\hline & \multicolumn{2}{|c|}{$\begin{array}{l}\text { Normal-weight } \\
\text { control group }\end{array}$} & \multicolumn{2}{|c|}{$\begin{array}{l}\text { Routine } \\
\text { antenatal care }\end{array}$} & \multicolumn{2}{|c|}{ Brochure } & \multicolumn{2}{|c|}{$\begin{array}{l}\text { Prenatal } \\
\text { sessions }\end{array}$} \\
\hline & mean & $\mathrm{SD}$ & mean & SD & mean & $\mathrm{SD}$ & mean & SD \\
\hline Height, cm & 179.85 & 5.89 & 178.92 & 7.83 & 180.08 & 8.94 & 180.53 & 8.47 \\
\hline Weight, kg & 83.24 & 10.96 & 88.43 & 16.73 & 86.62 & 16.27 & 89.66 & 14.39 \\
\hline BMI & 25.79 & 2.92 & 27.59 & 4.86 & 26.75 & 4.88 & 27.55 & 4.30 \\
\hline Weekly consumed candy servings & 3.48 & 3.44 & 5.64 & 3.87 & 4.85 & 3.91 & 3.94 & 2.45 \\
\hline Weekly consumed meat servings & 5.09 & 2.28 & 5.12 & 1.88 & 5.33 & 2.24 & 5.39 & 2.58 \\
\hline Weekly consumed fish servings & 0.87 & 0.61 & 0.90 & 0.71 & 1.15 & 0.83 & 1.15 & 0.70 \\
\hline Weekly consumed soft drink servings & 1.42 & 2.06 & 2.22 & 1.95 & 2.75 & 1.92 & 1.98 & 1.83 \\
\hline Weekly consumed water servings & 4.79 & 3.37 & 6.52 & 3.61 & 2.88 & 3.10 & 4.86 & 3.06 \\
\hline Weekly consumed milk servings & 5.02 & 3.45 & 5.96 & 3.05 & 4.62 & 3.58 & 5.01 & 3.64 \\
\hline Weekly consumed fruit servings & 5.63 & 3.81 & 4.28 & 3.88 & 4.08 & 5.87 & 4.77 & 3.97 \\
\hline Weekly consumed vegetables servings & 6.12 & 1.80 & 6.88 & 2.49 & 5.89 & 2.71 & 6.39 & 1.65 \\
\hline
\end{tabular}

to the prenatal session group. Subsequently, for each outcome for which the MANCOVA indicated no significant difference between the 3 intervention groups, the control group and the whole obese group were compared using one-way MANCOVA, while controlling for the children's age and sex, offspring birth weight and mothers' educational level.

\section{Results}

\section{Descriptive Statistics}

Characteristics of mothers, fathers and children in the control groups and the 3 intervention groups are shown in Tables $1-3$, respectively.

\section{The Effect of Lifestyle Interventions during Pregnancy}

MANCOVA indicated significant differences in the offspring CBQ surgency/extraversion $(F(2,82)=5.57, p<0.01)$ between the 3 intervention groups. Planned contrast analysis revealed that offspring CBQ surgency/extraversion was significantly lower in the brochure group than in both the group receiving routine antenatal care (contrast estimate $=-0.36, \mathrm{SE}=0.15, p=0.02$, $95 \% \mathrm{CI}[-6.66,-0.06]$ ) and the prenatal session group (contrast estimate $=-0.46, \mathrm{SE}=0.14, p<$ $0.01,95 \%$ CI [-0.74, -0.18]; Fig. 2). Planned contrast did not reveal any significant differences in offspring CBQ surgency/extraversion between the group receiving routine antenatal care and the prenatal session group ( $p>0.05)$. No significant group differences in offspring mental health, eating habits and anthropometric and cardiovascular measures were found.

\section{Comparison of the Whole Obese Group and the Normal-Weight Control Group}

The subsequent MANCOVA showed that children of mothers in the control group scored significantly lower on the SDQ emotional problems scale than children of mothers in the obese group $(F(1,156)=4.07, p<0.05)$. Furthermore, children of mothers in the control group also scored significantly lower on the SDQ externalizing problems scale $(F(1,156)=$ $6.10, p=0.02$ ) and trend-significantly lower on the SDQ internalizing problems scale $(F(1,156)=3.04, p=0.08)$. Children of mothers in the control group also scored significantly lower on the CBQ negative affectivity scale $(F(1,156)=5.42, p=0.02)$.

The latter MANCOVA indicated that children of mothers in the obese group had a significantly higher weight $(F(1,156)=12.22, p<0.01)$, resulting in a significantly higher BMI 
Braeken and Bogaerts: Effect of Lifestyle Interventions in Obese Pregnant Women on the Neurocognitive Development and Anthropometrics of Preschool Children

Table 3. Offspring characteristics

\begin{tabular}{|c|c|c|c|c|c|c|c|c|}
\hline & \multicolumn{2}{|c|}{$\begin{array}{l}\text { Normal-weight } \\
\text { control group }\end{array}$} & \multicolumn{2}{|c|}{$\begin{array}{l}\text { Routine } \\
\text { antenatal care }\end{array}$} & \multicolumn{2}{|l|}{ Brochure } & \multicolumn{2}{|l|}{$\begin{array}{l}\text { Prenatal } \\
\text { sessions }\end{array}$} \\
\hline & mean & SD & mean & SD & mean & SD & mean & SD \\
\hline SDQ prosocial behavior & 8.56 & 1.38 & 8.80 & 1.45 & 8.22 & 1.69 & 7.97 & 1.88 \\
\hline SDQ emotional problems & 1.71 & 1.76 & 2.07 & 1.66 & 1.96 & 2.10 & 2.36 & 2.22 \\
\hline SDQ hyperactivity & 3.26 & 2.56 & 3.27 & 2.46 & 3.30 & 2.58 & 4.10 & 2.93 \\
\hline SDQ conduct problems & 1.12 & 1.34 & 1.37 & 1.16 & 1.48 & 1.16 & 1.62 & 1.39 \\
\hline SDQ peer problems & 0.96 & 1.49 & 1.20 & 1.21 & 1.19 & 1.24 & 1.36 & 1.50 \\
\hline SDQ total difficulties & 7.05 & 5.08 & 7.90 & 4.29 & 7.93 & 5.40 & 9.44 & 5.10 \\
\hline SDQ externalizing problems & 2.83 & 2.27 & 3.43 & 2.24 & 3.44 & 2.79 & 3.97 & 2.51 \\
\hline SDQ internalizing problems & 2.68 & 2.59 & 3.27 & 2.13 & 3.15 & 3.05 & 3.72 & 3.15 \\
\hline CBQ surgency/extraversion & 4.25 & 0.62 & 4.30 & 0.58 & 4.00 & 0.56 & 4.40 & 0.52 \\
\hline CBQ negative affectivity & 3.76 & 0.83 & 4.09 & 0.73 & 3.83 & 0.80 & 4.03 & 0.76 \\
\hline CBQ effortful control & 5.35 & 0.73 & 5.57 & 0.69 & 5.31 & 0.65 & 5.34 & 0.62 \\
\hline Height, $\mathrm{cm}$ & 117.80 & 6.26 & 115.37 & 8.58 & 112.66 & 9.39 & 113.23 & 8.31 \\
\hline Weight, kg & 21.59 & 2.63 & 22.58 & 5.24 & 21.71 & 5.15 & 21.43 & 4.43 \\
\hline BMI & 15.52 & 1.01 & 16.75 & 1.90 & 16.88 & 2.01 & 16.55 & 1.95 \\
\hline Waist circumference, $\mathrm{cm}$ & 54.00 & 3.64 & 56.29 & 5.45 & 55.26 & 5.90 & 54.95 & 4.44 \\
\hline Hip circumference, cm & 56.75 & 3.49 & 58.47 & 5.86 & 57.37 & 6.14 & 57.21 & 4.92 \\
\hline Circumference at umbilicus level, $\mathrm{cm}$ & 55.38 & 3.75 & 58.02 & 6.10 & 56.00 & 7.50 & 56.67 & 5.36 \\
\hline Waist-to-hip ratio & 0.95 & 0.05 & 0.96 & 0.05 & 0.96 & 0.05 & 0.96 & 0.05 \\
\hline Waist-to-height ratio & 0.46 & 0.03 & 0.49 & 0.03 & 0.49 & 0.04 & 0.49 & 0.04 \\
\hline Heart rate, bpm & 85.70 & 10.09 & 86.17 & 14.10 & 87.59 & 12.82 & 88.54 & 8.78 \\
\hline Diastolic blood pressure, $\mathrm{mm} \mathrm{Hg}$ & 61.55 & 7.74 & 59.83 & 7.40 & 61.28 & 9.42 & 62.08 & 10.67 \\
\hline Systolic blood pressure, $\mathrm{mm} \mathrm{Hg}$ & 103.74 & 8.78 & 103.36 & 8.46 & 105.15 & 12.10 & 104.52 & 7.77 \\
\hline Weekly consumed candy servings & 4.34 & 3.36 & 3.78 & 2.37 & 4.37 & 2.63 & 3.83 & 2.17 \\
\hline Weekly consumed meat servings & 4.87 & 1.80 & 4.97 & 1.81 & 4.67 & 2.11 & 5.16 & 2.53 \\
\hline Weekly consumed fish servings & 0.97 & 0.90 & 1.03 & 0.68 & 1.25 & 0.68 & 0.97 & 0.57 \\
\hline Weekly consumed milk servings & 6.97 & 4.17 & 8.63 & 5.82 & 7.40 & 3.38 & 6.86 & 4.65 \\
\hline Weekly consumed fruit servings & 6.20 & 3.02 & 6.45 & 2.66 & 7.04 & 5.27 & 6.50 & 3.95 \\
\hline Weekly consumed vegetables servings & 6.18 & 2.19 & 6.25 & 2.32 & 6.70 & 2.91 & 6.33 & 2.39 \\
\hline Weekly consumed soft drink servings & 0.47 & 0.57 & 0.78 & 0.75 & 0.62 & 0.77 & 0.90 & 0.85 \\
\hline Weekly consumed water servings & 2.91 & 1.59 & 3.48 & 1.58 & 3.39 & 2.12 & 3.86 & 2.72 \\
\hline Age, years & 6.27 & 0.79 & 5.88 & 1.05 & 5.60 & 1.24 & 5.63 & 1.22 \\
\hline Sex & 0.52 & 0.50 & 0.63 & 0.49 & 0.48 & 0.51 & 0.56 & 0.50 \\
\hline Birth weight, g & $3,381.31$ & 508.69 & $3,551.00$ & 545.70 & $3,360.11$ & 575.89 & $3,379.83$ & 726.58 \\
\hline
\end{tabular}

$(F(1,156)=25.18, p<0.01)$. Additionally, there was also evidence for a significantly larger waist $(F(1,156)=12.57, p<0.01)$ and hip circumference $(F(1,156)=5.47, p=0.02)$ in children of mothers in the obese group. Relatedly, children of mothers in the obese group had a significantly higher waist-to-height ratio $(F(1,156)=20.81, p<0.01)$ and waist-to-hip ratio $(F(1,156)=4.23, p=0.04)$. Moreover, children of mothers in the obese group had a significantly larger circumference at umbilicus level $(F(1,156)=9.78, p<0.01)$. There were no significant differences in heart rate or blood pressure between the control group and the obese group $(p>0.1)$.

According to the MANOVA, children of mothers in the obese group tend to consume significantly more weekly servings of vegetables $(F(1,156)=4.06, p<0.05)$ and water $(F(1,156)=$ $6.43, p=0.01)$. Furthermore, children of mothers in the obese group consumed nearly significantly more weekly servings of fruit $(F(1,156)=3.43, p=0.07)$ and soft drink $(F(1,156)=$ $3.83, p=0.05)$. 
Braeken and Bogaerts: Effect of Lifestyle Interventions in Obese Pregnant Women on the Neurocognitive Development and Anthropometrics of Preschool Children

Fig. 2. Estimated marginal means of CBQ surgency/extraversion by intervention group.

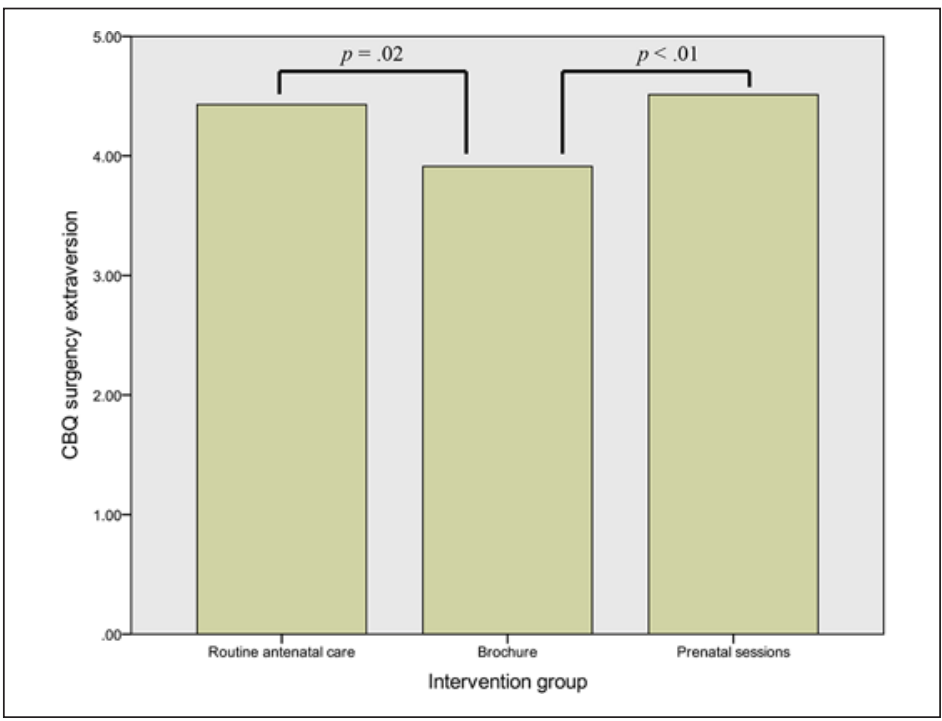

\section{Discussion/Conclusion}

The main result of this study is that children of obese mothers who received a brochurebased lifestyle intervention programme during pregnancy showed significantly less surgency/ extraversion compared to children of mothers who received routine antenatal care. Although there were no differences in offspring eating habits and anthropometric measures between the different intervention groups, the difference in surgency/extraversion requires attention, especially when considering previous research showing relationships between infant temperament and the development of obesity. More specifically, extraversion has been positively associated with overweight, possibly mediated by enjoyment of food and overeating habits in response to external cues $[27,28]$. Importantly, a recent study suggested that surgent infants only predicted increased weight gain during infancy when parents used food to soothe infant distress [29].

In contrast, the lifestyle interventions could not be associated with any significant differences in offspring mental health, eating habits and anthropometric and cardiovascular characteristics. Currently, it is unclear whether the lifestyle interventions during pregnancy might perhaps only have more short-term effects than long-term effects observable at the age of 3 years or older. It must be noted that all interventions only occurred during pregnancy. It might be important to have also follow-up interventions after pregnancy, as subjects may revert to earlier habits once intensive lifestyle intervention is finished. Remarkably, the prenatal session group, in contrast to the brochure group, did not show significant differences in offspring surgency/extraversion compared to the routine antenatal care group. This may also suggest that pregnant women receiving a more passive guidance in the form of a brochure might be more intrinsically motivated to change their lifestyle compared to pregnant women who participated in a more active prenatal session group with potentially more extrinsic motivation. Hence, it can also be hypothesized that pregnant women receiving more active guidance tend to revert to earlier habits much quicker than pregnant women receiving more passive guidance. Importantly, in our earlier RCT study, we reported a lower prevalence of women with excessive GWG in the brochure group (53.4 vs. $61.8 \%$ in the active prenatal session group) [23]. This difference might be a precursor of the observed differences in the children of the brochure and the active lifestyle intervention groups. This can be supported by some studies that report on an association between prenatal weight gain and negative outcome in the offspring in the longer term, such as autism spectrum disorders [30]. 
Braeken and Bogaerts: Effect of Lifestyle Interventions in Obese Pregnant Women on the Neurocognitive Development and Anthropometrics of Preschool Children

Additionally, other research shows that lifestyle interventions targeted at decreasing the risk of maternal gestational diabetes, preeclampsia or excessive fetal growth may be ineffective due to the fact that maternal pregravid and early pregnancy metabolic condition program early placenta function and gene expression [31]. These alterations in maternal/ placental function are known to occur in early pregnancy, possibly before the intervention was started. Another possible explanation is the key role that fathers have in the offspring eating habits and physical activity [32-34]. In the present study, many fathers of children from obese mothers had a remarkably high body weight, possibly moderating the effect of the lifestyle interventions [35]. Finally, future studies on the long-term effectiveness of lifestyle interventions during pregnancy could potentially benefit from a smaller age range when assessing the children.

When comparing the control group and the whole obese group, significant differences in offspring mental health were found. More specifically, children of mothers in the control group showed less emotional problems and internalizing and externalizing problems than children of mothers in the obese group. These results confirm the conclusion from an earlier review article stating that the offspring of women who are overweight or obese during pregnancy are at increased risk for externalizing problems and internalizing psychopathology in childhood $[8,36]$.

The results also show significant anthropometric differences between children of mothers in the obese group and children of mothers in the control group. Children of mothers in the obese group had a significantly higher weight and BMI, larger waist and hip circumference, higher waist-to-height ratio and waist-to-hip ratio and larger circumference at the umbilicus level. While an earlier study could not find significant differences in BMI and abdominal circumference between offspring of normal-weight mothers and offspring of obese mothers [37], our results are in line with other studies showing a link between maternal overweight and increased offspring's risk of obesity $[9,38]$. These differences show the relevance of studies investigating the potential positive effects of lifestyle interventions during pregnancy on the offspring. No evidence was found for any differences in heart rate or blood pressure between the control group and the obese group.

Our study has a number of strengths. To the best of our knowledge, it is the first study investigating the long-term effectiveness of lifestyle interventions during pregnancy. Furthermore, in contrast to other studies, the anthropometric measures, such as weight and height of mother and children, were not self-assessed but rated by the researchers. Standardized and validated measures and questionnaires were used. Markedly, almost $50 \%$ of the mothers who participated during pregnancy also participated when the children were 3-7 years old. Noticeably, the GWG of mothers who participated in the earlier RCT but declined to participate in the follow-up study was significantly lower. This may suggest that mothers who were not part of the follow-up study potentially benefited the most from the lifestyle intervention during pregnancy, possibly influencing the results of the present study. The children in our sample had relatively large age differences; we handled this inhomogeneity in the following ways. First, we used a validated age-adapted questionnaire, the CBQ [26], which is purposely designed to provide a detailed assessment of temperament in children 3-7 years of age. Second, in the multivariate analysis of covariance, child's age was one of the covariates.

While the present study did not find a positive effect of the lifestyle interventions during pregnancy on childhood obesity, the results suggest that the interventions can affect the offspring temperament by reducing surgency/extraversion. Future follow-up studies need to investigate how these temperament-related effects may influence obesity development later in life. 
Braeken and Bogaerts: Effect of Lifestyle Interventions in Obese Pregnant Women on the Neurocognitive Development and Anthropometrics of Preschool Children

\section{Acknowledgement}

The authors would like to thank Karolin Lagrin and Tinne Nuyts for their support in data collection.

\section{Statement of Ethics}

The authors state that all subjects have given their written informed consent and that the study protocol was approved by the institute's committee on human research.

\section{Disclosure Statement}

The authors have no conflicts of interest to declare.

\section{Funding Sources}

The authors received no financial support for the research, authorship and/or publication of this article.

\section{Author Contributions}

The first author was involved in the data collection, conducted the statistical analyses and wrote all sections of the manuscript. The second author was responsible for the data collection, study design and wrote the Introduction section.

\section{References}

1 Devlieger R, Benhalima K, Damm P, Van Assche A, Mathieu C, Mahmood T, et al. Maternal obesity in Europe: where do we stand and how to move forward?: A scientific paper commissioned by the European Board and College of Obstetrics and Gynaecology (EBCOG). Eur J Obstet Gynecol Reprod Biol. 2016 Jun;201:203-8.

2 World Health Organization. World Health Statistics. Geneva: WHO; 2012. Available from: https://www.who. int/gho/publications/world_health_statistics/2012/en/

3 Bogaerts A, Van den Bergh B, Nuyts E, Martens E, Witters I, Devlieger R. Socio-demographic and obstetrical correlates of pre-pregnancy body mass index and gestational weight gain. Clin Obes. 2012 Oct;2(5-6):150-9.

4 Garrido-Miguel M, Oliveira A, Cavero-Redondo I, Álvarez-Bueno C, Pozuelo-Carrascosa DP, Soriano-Cano A, et al. Prevalence of Overweight and Obesity among European Preschool Children: A Systematic Review and Meta-Regression by Food Group Consumption. Nutrients. 2019 Jul;11(7):E1698.

5 World Health Organization. Obesity and Overweight. Fact sheet. WHO; June 2016. Available from: http:// www.who.int/mediacentre/factsheets/fs311/en/

6 Institute of Medicine; Early Childhood Obesity Prevention Policies; Committee on Obesity Prevention Policies for Young Children; Birch LL, Parker L, Burns A, editors. Early Childhood Obesity Prevention Policies. Washington (DC): The National Academies Press; 2011.

7 Catalano PM, Shankar K. Obesity and pregnancy: mechanisms of short term and long term adverse consequences for mother and child. BMJ. 2017 Feb;356:j1.

8 Godfrey KM, Reynolds RM, Prescott SL, Nyirenda M, Jaddoe VW, Eriksson JG, et al. Influence of maternal obesity on the long-term health of offspring. Lancet Diabetes Endocrinol. 2017 Jan;5(1):53-64.

9 Leonard SA, Rasmussen KM, King JC, Abrams B. Trajectories of maternal weight from before pregnancy through postpartum and associations with childhood obesity. Am J Clin Nutr. 2017 Nov;106(5):1295-301.

10 Ludwig DS, Rouse HL, Currie J. Pregnancy weight gain and childhood body weight: a within-family comparison. PLoS Med. 2013 Oct;10(10):e1001521.

11 Gaillard R, Steegers EA, Franco OH, Hofman A, Jaddoe VW. Maternal weight gain in different periods of pregnancy and childhood cardio-metabolic outcomes. The Generation R Study. Int J Obes. 2015 Apr;39(4):677-85. 
Braeken and Bogaerts: Effect of Lifestyle Interventions in Obese Pregnant Women on the Neurocognitive Development and Anthropometrics of Preschool Children

12 Mamun AA, Mannan M, Doi SA. Gestational weight gain in relation to offspring obesity over the life course: a systematic review and bias-adjusted meta-analysis. Obes Rev. 2014 Apr;15(4):338-47.

13 Brion MJ, Zeegers M, Jaddoe V, Verhulst F, Tiemeier H, Lawlor DA, et al. Intrauterine effects of maternal prepregnancy overweight on child cognition and behavior in 2 cohorts. Pediatrics. 2011 Jan;127(1):e202-11.

14 Jo H, Schieve LA, Sharma AJ, Hinkle SN, Li R, Lind JN. Maternal prepregnancy body mass index and child psychosocial development at 6 years of age. Pediatrics. 2015 May;135(5):e1198-209.

15 Messerli-Bürgy N, Stülb K, Kakebeeke TH, Arhab A, Zysset AE, Leeger-Aschmann CS, et al. Emotional eating is related with temperament but not with stress biomarkers in preschool children. Appetite. 2018 Jan;120:256-64.

16 World Health Organization. The European Mental Health Action Plan 2013-2020. Copenhagen: WHO; 2015. Available from: http://www.euro.who.int/_data/assets/pdf_file/0020/280604/WHO-Europe-MentalHealth-Acion-Plan-2013-2020.pdf

17 Quinlivan JA, Julania S, Lam L. Antenatal dietary interventions in obese pregnant women to restrict gestational weight gain to Institute of Medicine recommendations: a meta-analysis. Obstet Gynecol. 2011 Dec;118(6):1395-401.

18 Tanentsapf I, Heitmann BL, Adegboye AR. Systematic review of clinical trials on dietary interventions to prevent excessive weight gain during pregnancy among normal weight, overweight and obese women. BMC Pregnancy Childbirth. 2011 Oct;11(1):81.

19 Thangaratinam S, Rogozinska E, Jolly K, Glinkowski S, Roseboom T, Tomlinson JW, et al. Effects of interventions in pregnancy on maternal weight and obstetric outcomes: meta-analysis of randomised evidence. BMJ. 2012 May;344(may16 4):e2088.

20 Dodd JM, Turnbull D, McPhee AJ, Deussen AR, Grivell RM, Yelland LN, et al.; LIMIT Randomised Trial Group. Antenatal lifestyle advice for women who are overweight or obese: LIMIT randomised trial. BMJ. 2014 Feb; 348:g1285.

21 Rogozińska E, Marlin N, Jackson L, Rayanagoudar G, Ruifrok AE, Dodds J, et al. Effects of antenatal diet and physical activity on maternal and fetal outcomes: individual patient data meta-analysis and health economic evaluation. Health Technol Assess. 2017 Aug;21(41):1-158.

22 Vesco KK, Leo MC, Karanja N, Gillman MW, McEvoy CT, King JC, et al. One-year postpartum outcomes following a weight management intervention in pregnant women with obesity. Obesity (Silver Spring). 2016 Oct;24(10):2042-9.

23 Bogaerts AF, Devlieger R, Nuyts E, Witters I, Gyselaers W, Van den Bergh BR. Effects of lifestyle intervention in obese pregnant women on gestational weight gain and mental health: a randomized controlled trial. Int J Obes. 2013 Jun;37(6):814-21.

24 Goodman R. The Strengths and Difficulties Questionnaire: a research note. J Child Psychol Psychiatry. 1997 Jul;38(5):581-6.

25 van Widenfelt BM, Goedhart AW, Treffers PD, Goodman R. Dutch version of the Strengths and Difficulties Questionnaire (SDQ). Eur Child Adolesc Psychiatry. 2003 Dec;12(6):281-9.

26 Sleddens EF, Kremers SP, Candel MJ, De Vries NN, Thijs C. Validating the Children's Behavior Questionnaire in Dutch children: psychometric properties and a cross-cultural comparison of factor structures. Psychol Assess. 2011 Jun;23(2):417-26.

27 Leung CY, Lumeng JC, Kaciroti NA, Chen YP, Rosenblum K, Miller AL. Surgency and negative affectivity, but not effortful control, are uniquely associated with obesogenic eating behaviors among low-income preschoolers. Appetite. 2014 Jul;78:139-46.

28 Leung CY, Miller AL, Kaciroti NA, Chen YP, Rosenblum K, Lumeng JC. Low-income pre-schoolers with higher temperamental surgency enjoy and respond more to food, mediating the path to higher body mass index. Pediatr Obes. 2016 Jun;11(3):181-6.

29 Stifter CA, Moding KJ. Infant temperament and parent use of food to soothe predict change in weight-forlength across infancy: early risk factors for childhood obesity. Int J Obes. 2018 Sep;42(9):1631-8.

30 Bilder DA, Bakian AV, Viskochil J, Clark EA, Botts EL, Smith KR, et al. Maternal prenatal weight gain and autism spectrum disorders. Pediatrics. 2013 Nov;132(5):e1276-83.

31 Catalano P, deMouzon SH. Maternal obesity and metabolic risk to the offspring: why lifestyle interventions may have not achieved the desired outcomes. Int J Obes. 2015 Apr;39(4):642-9.

32 Wake M, Nicholson JM, Hardy P, Smith K. Preschooler obesity and parenting styles of mothers and fathers: Australian national population study. Pediatrics. 2007 Dec;120(6):e1520-7.

33 Hall L, Collins CE, Morgan PJ, Burrows TL, Lubans DR, Callister R. Children's intake of fruit and selected energydense nutrient-poor foods is associated with fathers' intake. J Am Diet Assoc. 2011 Jul;111(7):1039-44.

34 Freeman E, Fletcher R, Collins CE, Morgan PJ, Burrows T, Callister R. Preventing and treating childhood obesity: time to target fathers. Int J Obes. 2012 Jan;36(1):12-5.

35 Dodd JM, Du Plessis LE, Deussen AR, Grivell RM, Yelland LN, Louise J, et al. Paternal obesity modifies the effect of an antenatal lifestyle intervention in women who are overweight or obese on newborn anthropometry. Sci Rep. 2017 May; $7(1): 1557$.

36 Van Lieshout RJ. Role of maternal adiposity prior to and during pregnancy in cognitive and psychiatric problems in offspring. Nutr Rev. 2013 Oct;71 Suppl 1:S95-101.

37 Tanvig M, Vinter CA, Jørgensen JS, Wehberg S, Ovesen PG, Beck-Nielsen H, et al. Effects of lifestyle intervention in pregnancy and anthropometrics at birth on offspring metabolic profile at 2.8 years: results from the Lifestyle in Pregnancy and Offspring (LiPO) study. J Clin Endocrinol Metab. 2015 Jan;100(1):175-83.

38 Catalano PM, Ehrenberg HM. The short- and long-term implications of maternal obesity on the mother and her offspring. BJOG. 2006 Oct;113(10):1126-33. 